\title{
Percepciones públicas, distancia psicológica y comunicación de riesgos frente al cambio climático: Una revisión contextualizada en Perú
}

\author{
Public perceptions, psychological distance and risk communication in the face of climate change: A \\ contextualised review in Peru
}

Fredy S. Monge Rodríguez ${ }^{1,2}$

\section{Resumen}

El cambio climático representa una gran amenaza para la humanidad y es motivo de preocupación para la comunidad científica, la sociedad y los políticos. La escasez de conocimientos contextualizados sobre la percepción de riesgo genera una brecha entre los países desarrollados y en vías de desarrollo, siendo estos últimos los más vulnerables a sus efectos. Todo esto nos lleva a plantear un estudio de revisión sobre percepción de riesgo, distancia psicológica y comunicación de riesgo frente al cambio climático, contextualizado en Perú. Los esfuerzos que se realizan desde diferentes instituciones públicas y organizaciones no gubernamentales son para proponer programas sólidos frente al cambio climático. En este contexto, el apoyo de políticas de gobierno contextualizadas en la realidad peruana resulta fundamental para poner un freno al incremento de temperatura y al aumento de emisiones de gases de efecto invernadero. Se concluye indicando la necesidad de estudios interdisciplinarios que permitan identificar la importancia del rol que juegan variables como la percepción de riesgo, distancia psicológica y las estrategias de comunicación en los procesos de adaptación y mitigación frente al cambio climático.

Palabras clave: percepción de riesgo, distancia psicológica, comunicación, cambio climático

\begin{abstract}
Climate change represents a major threat to humanity and it is a matter of concern for the scientific community, society and politicians. The lack of contextual knowledge on risk perception generates a gap between developed and developing countries, being the last ones the most vulnerable to its effects. All this leads us to propose a review study on risk perception, psychological distance and risk communication in the face of climate change, contextualised in Peru. Efforts made by different public institutions and non-governmental organizations are intended for proposing solid programs in the face of climate change. In this context, the support of government policies contextualized in the Peruvian reality is fundamental to put a brake on the increase in temperature and the increase in greenhouse gas emissions. Finally, the paper concludes by indicating the need for interdisciplinary studies that allow for the identification of the importance of the role played by variables such as risk perception, psychological distance and communication strategies in the processes of adaptation and mitigation to climate change.
\end{abstract}

Key words: risk perception, psychological distance, communication, climate change

\footnotetext{
Universidad Nacional de San Antonio Abad del Cusco. Cusco, Perú.

2 Centro de Investigación Ambiente, Comportamiento y Sociedad. Cusco, Perú.

ID ORCID: 0000-0002-9646-0161
} 


\section{INTRODUCCIÓN}

El cambio climático representa una de las principales amenazas que enfrenta la humanidad. De acuerdo al Panel Internacional sobre el Cambio Climático (IPCC, 2018) los efectos negativos sobre nuestro planeta cada día van en aumento y se pueden observar a través de la presencia de peligros naturales como los eventos extremos, avalanchas, tormentas, las olas de calor e inundaciones, poniendo en riesgo nuestros sistemas económicos, sociales, de salud y la vida de las personas, como lo indica el reporte del Panel Internacional sobre el Cambio Climático. Además, el reporte precisa que "los riesgos relacionados con el clima para los sistemas naturales y humanos son mayores con un calentamiento global de $1,5{ }^{\circ} \mathrm{C}$ que los que existen actualmente". (p. 7). Debido a los peligros que implica para nuestra sociedad, es importante centrar nuestra atención en los impactos de estos riesgos desde el campo de las ciencias sociales (Biresselioglu et al., 2020). Un reciente informe del IPCC el año 2019, elaborado por expertos que conforman el Panel Intergubernamental de Cambio Climático (IPCC), pone en evidencia que la práctica de estilos de vida sostenibles y los cambios de comportamientos pro ambientales, son las mejores opciones que tenemos hoy en día para poner un límite al incremento de las temperaturas, que, como sabemos, representan una de las principales causas del cambio climático. Sin embargo, el cambio climático también podría ser percibido como un fenómeno distante, por ejemplo, cuando algunas personas tienden a distanciar o alejar los riesgos mentalmente, aunque este comportamiento los exponga a riesgos mayores (Kim \& Wolinsky-Nahmias, 2014).

Los informes previos del IPCC (2007), basados en una profunda revisión de literatura científica, dejaron en evidencia que el incremento desproporcionado de temperatura en nuestro planeta se manifiesta de manera desigual en nuestro planeta. Estos informes también precisan que la mayor parte del aumento de las temperaturas reportadas en los promedios globales, analizados desde mediados del siglo XX, son de origen antropogénico, en donde las actividades del hombre han contribuido en el incremento de las emisiones de gases de efecto invernadero, como la quema de combustibles fósiles, actividades como la producción de energía y las actividades que promueven la deforestación global. Por otro lado, las políticas de gobiernos hasta el momento resultan ineficientes, y no demuestran grandes esfuerzos para reducir las emisiones de carbón (Compston \& Bailey, 2008).
Además, se debe reconocer que la comunicación de riesgos es una variable determinante para la toma de conciencia y desarrollo de políticas de adaptación y mitigación frente al cambio climático, esto a su vez conlleva un compromiso y apoyo de las políticas públicas, que hasta la fecha no tienen el impacto esperado (Filho, 2019).

\section{Iniciativas políticas frente al cambio climático en Perú}

Como sabemos, gran parte de la economía de Perú se basa en sus abundantes recursos naturales y en una actividad industrial constante sobre todo a nivel de la industria minera (Triscritti, 2013). Sin embargo, Perú se encuentra entre los países más vulnerables a los desastres naturales en América Latina (Carey, 2012), a pesar que su contribución en la generación de gases de efecto invernadero es mínima en comparación con otros países. Por otro lado, resulta paradójico que los países que emiten mayor cantidad de gases de efecto invernadero sean los menos afectados por los impactos directos del calentamiento global (Samson et al., 2011). Bajo este panorama, Perú ha propiciado algunas acciones y compromisos políticos en el tema de cambio climático, una de las primeras acciones fue la elaboración de la propuesta de la agenda política durante el año de 1990, gracias a la cooperación que venía desarrollando con otras organizaciones internacionales.

Otro hito importante es la creación de la Ley Orgánica de Gobiernos Regionales (Ley N. ${ }^{\circ}$ 27867) en el año 2002, esta ley tuvo por objetivo descentralizar la normativa ambiental y solicitar la formulación de una Estrategia Regional de Cambio Climático, con temas de adaptación y mitigación. También tenemos la primera Estrategia Nacional de Cambio Climático, que entró en vigor en 2003. Perú también cuenta con el Proyecto de Planificación ante el Cambio Climático, que tiene el propósito de explorar la viabilidad de evaluar la reducción de emisiones de carbono. Además, cuenta con el Plan de Gestión de Riesgos y Adaptación al Cambio Climático en el Sector Agrario 2012-2021. Un evento trascedente en la política de cara al cambio climático fue el evento de la $20^{\mathrm{a}}$ Conferencia de las Partes de la Convención Marco de las Naciones Unidas sobre el Cambio Climático (CMNUCC-COP20) auspiciada por Perú en el 2014, que pasó a ser un evento político en la historia de la necesidad de tomar decisiones sobre cambio climático en Perú (UNESCO, 2014). Esta convención permitió al gobierno mostrar el trabajo que se está realizando en el país y colocar 
en la agenda política global cuestiones de importancia para el país y la región latinoamericana. Perú identificó en la reunión COP20 la oportunidad para proponer el tema del cambio climático. Sin embargo, los esfuerzos nacionales se han centrado en la mitigación, dejando de lado los estudios sobre percepción y comunicación del riesgo, que resultan indispensables, para el diseño de estrategias de adaptación y mitigación (Monge et al., 2019).

\section{Percepción del riesgo, distancia psicológica y cambio climático}

Los riesgos que implica el cambio climático, nos muestran una serie de desafíos a nivel de todo el mundo (Swim et al., 2011). Para un mejor entendimiento del problema es necesario identificar algunos factores como la forma de pensar o actuar y que en muchas ocasiones cambian, debido a situaciones geográficas, políticas y económicas; estos elementos juegan un rol importante en relación a cómo una persona percibe públicamente una determinada situación como un riesgo, que requiere atención urgente. Algunos trabajos ponen de manifiesto que la naturaleza de la percepción humana implica las interacciones de factores cognitivos, sociales, culturales y emocionales, estos procesos interactuando de manera conjunta nos permiten poder diferenciar entre las amenazas del mundo real y la forma subjetiva de experimentar esas amenazas (Pidgeon et al., 2003). Como lo menciona el investigador Sjoberg (2000) la percepción de riesgo se construye en la mente a partir de diferentes experiencias. En esta misma línea, Slovic (1992) señala que el concepto de riesgo es algo que ha sido creado por los seres humanos, y por tal razón, no hay riesgo independiente de la mente y la cultura. Por lo tanto, los impactos negativos del cambio climático, deben ser analizados a partir de percepciones individuales o grupales, identificando los elementos de riesgo que considere una población, que podrían variar de una cultura a otra. Por citar un ejemplo, en el Reino Unido el cambio climático es considerado como un tema prioritario; de acuerdo a las declaraciones del actual primer ministro Johnson "estamos exigiendo una política ambiciosa de todos los departamentos para garantizar que los hogares, las empresas, la industria, el transporte y la tierra ayuden a conseguir el carbono neto cero" (Harvey, 2019). A pesar de la gravedad del tema, en algunos lugares el cambio climático ha sido percibido como un problema que no amerita mucha atención y en consecuencia sus decisiones políticas son menos estables, como es el caso de Estados Unidos y China (Leiserowitz et al., 2014). Además, se observa que la percepción del cambio climático no solamente puede variar de un país a otro, sino también varía de un individuo a otro, en un mismo país o incluso ciudad (Smith \& Leiserowitz, 2014).

En este sentido, entender el cambio climático, desde el enfoque de la percepción de riesgo, puede resultar una expresión poco común para los individuos y sociedades que han experimentado sus impactos (Breakwell, 2010). Este fenómeno está representado por experiencias únicas, debido a que la magnitud y la escala son particulares para cada individuo y no se determinan a través de escalas de tiempo para la identificación de los cambios en el clima; por el contrario, las ciencias naturales sí consideran los períodos de tiempo que van de 25 a 30 años, para identificar los cambios en el clima. Por otro lado, desde la perspectiva social el cambio climático es percibido como un proceso lento, acumulativo, y en gran parte no observable para la mayoría de individuos con excepción de los expertos; por lo tanto, no podría ser experimentado de manera directa, sino a través de sus efectos secundarios (Weber, 2010). Por ejemplo, en el caso de la disminución de los recursos hídricos debido al retroceso de los glaciares, el individuo no percibe el calentamiento global como causa del cambio climático, sino centra su atención en la diminución del agua, producto del retroceso glaciar, recurso que es indispensable para sustentar su actividad económica que depende de la agricultura. El tema aún se vuelve más complejo y al mismo tiempo interesante, cuando pretendemos comprender e interpretar a nuestros antepasados que estuvieron más expuestos a las amenazas climáticas, y que contaban con menos recursos de mitigación, a diferencia de los que hoy se tiene (Gifford, 2011).

Sin embargo, existen ocasiones en que el cambio climático es percibido como algo que no es necesario priorizar por el momento, es un problema que se encuentra muy distante (Kim \& Wolinsky-Nahmias, 2014). Al respecto, diversos trabajos han demostrado que en algunas situaciones las personas pueden alejar los riesgos mentalmente, ubicándolos de una forma que estén muy distantes a ellos (Lorenzoni \& Pidgeon, 2006); por ejemplo, si un individuo percibe el cambio climático como algo lejano geográficamente, piensa que está lejos de la ubicación donde se encuentra y que este fenómeno solo impactará en otros lugares y en, consecuencia, no se verá afectado. Por otro lado, también están los individuos que tienden a distanciar estos riesgos a través del tiempo, en este caso pueden percibir que estos riesgos no tienen probabilidad de 
suceder en un futuro inmediato o sea en el presente, sino por el contrario, piensan que sucederán en el futuro, muchos años más adelante, y por lo tanto esto no les afectará. Para una explicación más detallada vemos necesario describir la Teoría de Niveles de Constructos o Construal Level Theory (CLT, por sus siglas en inglés) que fue propuesta por Liberman y Trope (2008). Esta teoría establece cuatro dimensiones que son las determinantes de la distancia psicológica: a) distancia espacial o geográfica; b) distancia temporal; c) distancia entre el perceptor y el lugar de ubicación y d) distancia de un objetivo social, es decir, de otro individuo o grupo.

La distancia psicológica vinculada al cambio climático ha sido motivo de diversos estudios, como el deMilfont (2010), quien precisa que el cambio climático se percibe como distante en todas estas dimensiones; por tal razón, sugiere que deban desarrollarse estudios que tengan el propósito de identificar el potencial que puede tener la manipulación de la distancia percibida, y que a través de las evidencias de estudios experimentales se puedan promover estrategias para implementar cambios de comportamientos sostenibles en favor del medio ambiente. Además, la CLT propone que los eventos psicológicamente distantes se representen mediante constructos abstractos que presentan características generales descontextualizadas, mientras que los eventos psicológicamente cercanos se representan mediante constructos concretos de bajo nivel compuestos por detalles contextuales específicos. Además, existen investigaciones que han dejado en evidencia que las personas que perciben y son conscientes del cambio climático tienen más probabilidades de pensar que los impactos se darán en personas y lugares geográfica y temporalmente lejanos de ellos (Leiserowitz et al., 2010). Asimismo, los impactos del cambio climático tienden a considerarse más graves para los lugares distantes (Spence \& Pidgeon, 2010). Se ha demostrado también que las personas distinguen claramente entre los efectos personales y sociales del cambio climático, y en varios estudios se ha observado que los riesgos personales del cambio climático se consideran inferiores a los riesgos sociales (Leiserowitz et al., 2010).

Estos resultados son encontrados de manera similar en Perú (Monge et al., 2019). En primer lugar, se encuentra la percepción de amenaza que representa el cambio climático para el medio ambiente, el 79\% percibió como grave y muy grave esta amenaza. En segundo lugar, se encuentra la preocupación por los impactos del cambio climático en el Perú, el 77\% indicó que se encuentra preocupado y muy preocupado. Seguidamente, se encuentra la preocupación sobre las consecuencias del cambio climático para todo el mundo, el $76 \%$ se encuentra preocupado y muy preocupado. Luego se observa que el $74 \%$ de los encuestados, se encuentran muy preocupados de que el cambio climático amenace su salud. Vemos también que $66 \%$ indica que se encuentra preocupado o muy preocupado de que el cambio climático represente una amenaza a nivel personal. Seguidamente, vemos que un $64 \%$, se encuentra preocupado o muy preocupado por el cambio climático. Por último, el 54\%, indicó que se encuentra preocupado o muy preocupado respecto a las consecuencias que implica el cambio climático. Al respecto, se reportan hallazgos similares en Estados Unidos y Europa (Lee et al., 2015). Sin embargo, es importante resaltar los niveles de impactos que el cambio climático podría tener en países en desarrollo, en particular en los situados en el sur geográfico, lo cual resulta preocupante, debido a que en su mayoría son países que no tienen suficientes recursos y capacidad para enfrentar todos los impactos negativos del cambio climático.

En consecuencia, las diferencias identificadas en los niveles de percepción de riesgo del cambio climático nos ayudan a identificar el sentido en el cual deberían estar orientadas las acciones de apoyo político en Perú, y de esta forma proponer acciones direccionadas en estas áreas. Sin embargo, en la actualidad aún no hemos llegado a un nivel de entendimiento y consenso entre los científicos y los políticos respecto a la toma de decisiones, decisiones que deben estar soportadas en datos objetivos y que deben generar propuestas o programas políticos que tengan por finalidad fortalecer la comunicación de riesgos y de esta manera concientizar a las poblaciones de los riesgos que implica el cambio climático, una vez conseguido este objetivo se debe iniciar los programas de adaptación y mitigación frente al cambio climático.

\section{Avances en psicología sobre cambio climático y percepción de riesgos}

El acuerdo de París, firmado el año 2015, contó con la presencia de diversos líderes políticos, quienes se reunieron en la Conferencia de las Naciones Unidas sobre el Cambio Climático. En esta conferencia, cada uno de los líderes se comprometieron a incluir elementos sociales vinculados al cambio climático. Lo cual comprende un momento histórico para el fortalecimiento y desarrollo de las líneas 
de investigación desde las diferentes áreas del comportamiento; en esta; misma línea, los informes recientes del Grupo Intergubernamental de Expertos sobre el Cambio Climático (IPCC, 2018, 2019), colocan en primer plano el importante rol que juegan los comportamientos y las acciones sociales, en la disminución del incremento de la temperatura mundial a $1,5^{\circ} \mathrm{C}$, o incluso $2^{\circ} \mathrm{C}$. Sin embargo, nos llama la atención que las ciencias del comportamiento, hasta la fecha no hayan podido generar propuestas serias que respondan a las particularidades de cada contexto, solo a través de estos aportes se podrían acortar las brechas de conocimiento.

Algo que debemos reconocer, es que hoy en día la psicología como disciplina científica ha desarrollado y también viene empleando diferentes metodologías que responden a toda la rigurosidad científica y que aportan al desarrollo de un cuerpo de conocimientos que permitan tener un mejor entendimiento del cambio climático. En este proceso se han desarrollados tres campos importantes (Clayton et al., 2015):

- Primero, las percepciones públicas del cambio climático.

- Segundo, el manejo del comportamiento humano con relación al cambio climático y las respuestas de mitigación.

- Tercero, el estudio de los impactos del cambio climático sobre el bienestar humano y las respuestas de adaptación.

Además, como ya lo ha indicado la comunidad científica, uno de los factores determinantes que propician e impulsan la adaptación y la mitigación en relación a los impactos del cambio climático en el ámbito poblacional, es la percepción de riesgo (Dai et al., 2015). En este contexto, la psicología, como ciencia encargada del estudio del comportamiento y los procesos mentales, tiene la tarea de generar un mayor bagaje de aportes científicos que impulsen la implementación de estrategias de comunicación en alianza con los tomadores de decisiones, para la promoción de comportamientos de adaptación y mitigación que se encuentren dirigidos a la reducción de los efectos negativos del cambio climático, sobre todo en las poblaciones de mayor vulnerabilidad (Manning \& Clayton, 2018).

Analizar factores psicológicos relacionados a la percepción del riesgo también es importante para los ciudadanos, ya que permite visualizar el apoyo de las medidas políticas del clima, algo que es particularmente relevante para Perú, debido a que forma parte de su estrategia política para hacer frente al cambio climático. Este lineamiento político se encuentra aprobado mediante Decreto Supremo $\mathrm{N}^{\mathrm{O}}$ 058-2016-RE, que ratifica la participación de Perú en el Acuerdo de París (Ministerio del Ambiente, 2016), el cual plantea llegar a cero en las emisiones netas de gases de efecto invernadero antes del año 2100. Para este fin, se deben de poner en marcha diferentes acciones orientadas a la promoción de medidas de mitigación y adaptación, lideradas bajo un principio de responsabilidades comunes pero diferenciadas entre los científicos, las instituciones, tomadores de decisiones y ciudadanos; del mismo modo este acuerdo compromete a los países que forman parte del mismo a mantener el aumento de temperatura debajo de $2^{\circ} \mathrm{C}$, ambicionando la meta de $1.5^{\circ} \mathrm{C}$.

Por esta razón, resulta indispensable que, a través de las ciencias de la conducta, se analicen las consecuencias del cambio climático, y cómo estas influyen sobre los individuos y la sociedad. Además, una cantidad considerable de investigaciones reportan que la percepción del riesgo es un predictor importante sobre la voluntad pública, la toma de decisiones y las estrategias de comunicación, que son elementos importantes que ayudan en la disminución de los efectos negativos del cambio climático (Leiserowitz, 2006; Tobler et al., 2012).

Bajo este panorama asumimos que, para un mejor entendimiento de los complejos vínculos entre la psicología de la percepción del riesgo del cambio climático y la toma de decisiones, es necesario analizar estos tópicos a partir de modelos que incluyan variables psicológicas, sociales y culturales. Estos modelos, más allá del beneficio de su aporte teórico en un determinado campo del conocimiento, permiten tener una visión integral de un determinado fenómeno, en este caso la percepción de riesgo del cambio climático. Además, nos brindan elementos para los tomadores de decisiones debido a que permiten a las autoridades desarrollar estrategias de comunicación para involucrar a las comunidades con acciones más efectivas reflejadas en planes de mitigación y adaptación, para hacer frente a las consecuencias del cambio climático y sus impactos en la salud humana.

\section{Modelo psicológico de la percepción de riesgo del cambio climático}

Los modelos psicológicos de percepción de riesgos asociados al cambio climático en años 
pasados incluyeron el estudio de variables cognitivas, emocionales o socio culturales de forma separada. Recientemente estudios como los del investigador van der Linden (2015) han reportado resultados que explican la percepción de riesgo de manera integrada. Por otro lado, es evidente que estos trabajos son más frecuentes en países desarrollados, siendo también necesarios en países en vías de desarrollo debido a su alta vulnerabilidad frente a los impactos negativos del cambio climático. Ese es el caso de Perú, y hasta donde sabemos existen escasos estudios que estén orientados a adaptar estos modelos a una determinada realidad, como es el caso de los países sudamericanos (Brügger et al., 2016). La adaptación de modelos sobre percepción de riesgos, permite avanzar y fortalecer las estrategias de comunicación de riesgos. La propuesta considerada en esta revisión plantea un modelo teórico integrado de la percepción del riesgo frente al cambio climático, que combina cuatro dimensiones teóricas: variables cognitivas, experienciales, socioculturales y sociodemográficas. Este modelo es conocido como Modelo de Percepción del Riesgo del Cambio Climático (Climate change risk perception model o, CCRPM, por sus siglas en inglés), propuesto por van der Linden (2015).

\section{Comunicación del riesgo y toma de decisiones políticas.}

El estudio de la comunicación de riesgos vinculados al cambio climático es indispensable debido a que su puesta en práctica ayuda a generar mayor conciencia de peligro en las poblaciones, esto a la vez impulsa la movilización de diferentes acciones individuales o grupales para hacer frente a los impactos negativos. Las acciones que vienen desarrollando el IPCC desde el año 1998 y el Ministerio del Ambiente en Perú, para buscar y comprometer el apoyo del público y los políticos, para enfrentar las casusas del cambio climático a través de la comunicación de riesgos, han tenido poco efecto (Filho, 2019). En este sentido, los trabajos realizados desde el campo de las ciencias sociales y la toma de decisiones, precisan que la comunicación de riesgos podría traer algunas dificultades en su proceso, como la manera de interpretarse; por ejemplo, cuando se trata de expertos en el clima o legos en el tema, debido a que ambos tienen diferentes perspectivas por el tipo de conocimiento que tienen, dificultando la comunicación de riesgo (Fischhoff, 1995). Además, existen otras barreras en la comunicación de riesgos, más aún cuando se trata de una cuestión política que implica la comunicación de contenidos técnicos y que en muchas ocasiones limita la posibilidad de que la población en general entienda el mensaje. Por otro lado, se han identificado algunos factores que ayudan en la comunicación de riesgos como son, el peligro y la indignación, que cuando están vinculados con el contexto cultural y económico, podrían motivar a los individuos a adherirse a los mensajes y aceptar las estrategias de comunicación propuestas (Malecki et al., 2020). En caso de Perú, se ha avanzado muy lento en el diseño de estrategias de comunicación de riesgos, a pesar que se observa un esfuerzo de algunas organizaciones no gubernamentales e instituciones públicas, vemos que a la fecha no son suficientes. Sin embargo, debemos considerar dos aspectos relevantes en la comunicación de riesgos, primero las campañas desarrolladas en Perú son complejas por su diversidad cultural, y los resultados no son satisfactorios, a pesar que se cuenta con recursos suficientes para su ejecución. En segundo lugar, se indica que la mayoría de campañas se han centralizado solo en Lima, dejando de lado la propuesta de campañas que respondan a las necesidades a nivel regional y/o local (Takahashi \& Martínez, 2017). Frente a esta realidad, la literatura científica propone desarrollar un acercamiento estratégico para la comunicación del cambio climático; este acercamiento estratégico está conformado por dos elementos, el primero es la escucha estratégica y el segundo es la organización estratégica, ambas deben ser analizadas, diseñadas, implementadas y evaluadas de manera estratégica (Pidgeon \& Fischhoff, 2011).

Respecto a la primera estrategia, es necesario desarrollar herramientas de escucha activa, con la participación de científicos, políticos y población en general. Solo de esta manera se puede identificar si están o no teniendo efectos los mensajes de los expertos y si realmente los políticos están cumpliendo su parte, de dar el adecuado soporte logístico y económico a estos programas. Esta estrategia de condición debe estar basada en los aportes de las ciencias sociales y estudios sólidos sobre toma de decisiones, que requerirán de una implementación rigurosa y una evaluación empírica para determinar su eficacia, pero como podemos observar hasta la fecha estas evaluaciones son extremadamente escasas (Pidgeon \& Fischhoff, 2011). Sin embargo, de acuerdo a los autores citados anteriormente, sabemos que la gran mayoría de estrategias de comunicación se encuentran basadas en nociones intuitivas sobre qué decir y cómo decirlo. En relación a la segunda, que es la organización estratégica, precisan que, para una óptima comunicación del cambio climático, es necesario considerar contribuciones sostenidas a través de los equipos de investigación interdisciplinarios, que 
trabajen dentro de una política y un marco institucional que den soporte sólido a sus esfuerzos. Los equipos de científicos deberían estar conformados por diferentes grupos de expertos, como climatólogos, especialistas en temas de adaptación y mitigación, especialistas en toma de decisiones, científicos sociales, comunicadores y diseñadores de campañas de comunicación. Una vez reunidos, estos equipos deben coordinar para que los expertos orienten su atención en los procesos para mejorar los procesos de comunicación de riesgos.

En suma, podemos indicar acerca de la comunicación y el cambio climático, que hasta el momento existe frustración por parte de los científicos de tener respuestas muy limitadas por parte de los políticos y la población, frente a la grave crisis que enfrenta el planeta. Nos queda la tarea de poner en práctica estrategias basadas en sólidos principios sociales, que estén acompañados de una rigurosa evaluación empírica.

\section{CONCLUSIONES}

Los aportes examinados en el presente trabajo son útiles en la generación de conocimientos en el marco del cambio climático y a la vez nos permiten evaluar las implicancias para Perú. Como se ha podido observar, los aportes generados desde la percepción del riesgo revelan predictores importantes sobre la voluntad pública y la toma de decisiones, a pesar de que la distancia psicológica podría representar un problema y dificultar la comunicación de riesgos, en la tarea de poner un freno a los impactos negativos del cambio climático. Desde las ciencias del comportamiento nos quedan algunos retos, como impulsar la práctica de comportamientos pro ambientales, para luchar contra el incremento de la temperatura en nuestro planeta que responde a causas de origen antropogénico. Además, estos aportes también deben contribuir a la toma de decisiones a nivel político. Es evidente que las decisiones políticas tienen un rol determinante en este escenario, pero al menos en Perú han sido hasta la fecha ineficientes, y tampoco se demuestran esfuerzos para implementar mejoras en las estrategias de afrontamiento al cambio climático, sobre todo en el tema de la comunicación de riesgos. Futuros estudios deben promover investigaciones interdisciplinarias, que evalúen y determinen los factores de la percepción de riesgo, distancia psicológica y sus vínculos con la comunicación de riesgos en poblaciones vulnerables, y que del mismo modo permitan identificar los factores psicológicos, culturales, medios de vida y económicos implicados, para, de esta manera, promover los programas de adaptación y mitigación frente al cambio climático.

Declaración de conflicto de intereses: El autor declara que el presente artículo no tiene conflicto de intereses.

Contribución del autor: Por ser un único autor, el trabajo ha sido completamente realizado por el autor.

\section{REFERENCIAS BIBLIOGRÁFICAS}

Biresselioglu, M., Demir, M., Solak, B., Kayacan, A., \& Altinci, S. (2020). Investigating the trends in arctic research: The increasing role of social sciences and humanities. Science of The Total Environment, 729, 139027.https://doi.org/10.1016/j.scitotenv.2020.1390 27

Breakwell, G. M. (2010). Models of risk construction: Some applications to climate change. WIREs: Climate Change, 1(6), 857-870.

Brügger, A., Tobias, R., \& Monge, F. S. (Diciembre, 2016). A replication and extension of the sociopsychological model of climate change risk perceptions. Trabajo presentado en la 2016 Annual Meeting, Society for Risk Analysis. San Diego, CA. Resumen recuperado de http://www.sra.org/sites/ default/files/pdf/events/SRA\%20Final $\% 20$ Program\%20-\%20R6.pdf

Carey, M., Huggel, C., Bury, J., Portocarrero, C., \& Haeberli, W. (2012). An integrated socioenvironmental framework for glacier hazard management and climate change adaptation: lessons from Lake 513, Cordillera Blanca, Peru. Climatic Change, 112, 733-767. http://doi.org/10.1007/ s10584-011-0249-8

Clayton, S., Devine-Wright, P., Stern, P. C., Whitmarsh, L., Carrico, A., Steg, L., \& Bonnes, M. (2015). Psychological research and global climate change. Nature Climate Change, 5(7), 640-646. https://doi. org $/ 10.1038 /$ nclimate2622

Compston, H., \& Bailey, I. (2008). Turning down the heat: the politics of climate policy in affluent democracies. Palgrave Macmillan.

Dai, J., Kesternich, M., Löschel, A., \& Ziegler, A. (2015). Extreme weather experiences and climate change beliefs in China: An econometric analysis. Ecological Economics, 116, 310-321. https://doi. org/10.1016/j.ecolecon.2015.05.001

Filho, L.W. (2019). An overview of the challenges in climate change communication across various audiences. En L. W. Filho, B. Lackner, \& H. McGhie (Eds.), Addressing the challenges in communicating climate change across various audiences (pp. 1-11). Springer. 
Fischhoff, B. (1995). Risk perception and communication unplugged: 20 years of process. Risk Analysis, 15, 137-145.

Gifford, R. (2011). The dragons of inaction: Psychological barriers that limit climate change mitigation and adaptation. American Psychologist, 66 (4), 290-302.

Harvey, F. (2019, 18 de diciembre). Boris Johnson urged to take world lead on climate action. The Guardian. https://www.theguardian.com/environment/2019/ $\mathrm{dec} / 18 /$ boris-johnson-urged-to-take-world-lead-onclimate-action

Intergovernmental Panel on Climate Change (IPCC). (2007). 4th Assessment Report. Cambridge University Press.

Intergovernmental Panel on Climate Change (IPCC). (2018). Global warming of $1.5^{\circ} \mathrm{C}$.: Author.

Intergovernmental Panel on Climate Change (IPCC). (2019). Climate change and land. Author.

Kim, S. Y., \& Wolinsky-Nahmias, Y. (2014). Crossnational public opinion on climate change: The effects of affluence and vulnerability. Global Environmental Politics, 14(1), 79-106. https://doi.org/10.1162/ GLEP_a_00215

Lee, T., Markowitz, E., Howe, P., Ko, C.-Y. \& Leiserowitz, A. A. (2015). Predictors of public climate change awareness and risk perception around the world. Nature Climate Change 5, 1014-1020. https://doi.org/10.1038/nclimate2728

Leiserowitz, A. (2006). Climate change risk perception and policy preferences: The role of affect, imagery and values. Climatic Change, 77, 45-72.

Leiserowitz, A., Maibach, E., Roser-Renouf, C., \& Smith, N. (2010). Climate change in the American Mind: Americans' global warming beliefs and attitudes in January 2010. Yale University and George Mason University. Yale Project on Climate Change Communication.

Leiserowitz, A., Maibach, E., Roser-Renouf, C., Feinberg, G., \& Rosenthal, S. (2014). Climate change in the American mind April 2014. Yale Project on Climate Change Communication, Yale University

Liberman, N., \& Trope, Y. (2008). The psychology of transcending the here and now. Science, 322, 12011205.

Lorenzoni, I., \& Pidgeon, N. (2006). Public views on climate change: European and USA perspectives. Climatic Change, 77, 73-95.

Malecki, K., Keating, J., \& Safdar, N. (2020). Crisis communication and public perception of COVID-19 risk in the Era of Social Media. Clinical Infectious Disease, 20, 1-6. https://doi.org/10.1093/cid/ciaa758

Manning, C., \& Clayton, S. (2018). Threats to mental health and wellbeing associated with climate change. En S. Clayton \& C. Manning (Eds.), Psychology and climate change: Human perceptions, impacts, and responses (pp. 217-244). Elsevier.
Milfont, T. L. (2010). Global warming, climate change and human psychology. En V. Corral-Verdugo, C. H. Garcia-Cadana \& M. Frias-Arment (Eds.), Psychological approaches to sustainability: Current trends in theory, research and practice (pp. 19-42). Nova Science.

Ministerio del Ambiente. (2016). Perú ratifica el Acuerdo de París y consolida su liderazgo climático mundial. https://www.minam.gob.pe/somoscop20/2016/07/22/ peru-ratifica-el-acuerdo-de-paris-y-consolida-suliderazgo-climatico-mundial/

Monge, F.S., Tobias, R., \& Brügger, A. (2019). Cambio climático y percepciones en comunidades quechuahablantes. Alpha.

Organización de las Naciones Unidas para la Educación, la Ciencia y la Cultura. (2014). Participación de la UNESCO en la Conferencia sobre Cambio Climático de las Naciones Unidas 2014 (COP 20). http://www. unesco.org/new/es/natural-sciences/special-themes/ global-climate-change/cop/lima-climate-changeconference-cop-20/

Pidgeon, N., \& Fischhoff, B. (2011). The role of social and decision sciences in communicating uncertain climate risks. Nature Climate Change, 1, 35-41. http:/ doi.org/10.1038/nclimate1080

Pidgeon, N. F., Kasperson, R. E., \& Slovic, P. (2003). The social amplification of risk. Cambridge University Press.

Takahashi, B., \& Martinez, A. (2017). Climate change communication in Peru. Oxford Research Encyclopedia of Climate Science. USA. http://doi. org/10.1093/acrefore/9780190228620.013.574

Tobler, C., Visschers, V. H. M., \& Siegrist, M. (2012). Addressing climate change: Determinants of consumers' willingness to act and to support policy measures. Journal of Environmental Psychology, 32(3), 197-207.

Triscritti, F., (2013). Mining, development and corporatecommunity conflicts in Peru. Community Development Journal, 48(3), 437-450. https://doi.org/10.1093/cdj/ bst024

Samson, J., Berteaux, D., McGill, D., \& Humphries, M. (2011). Geographic disparities and moral hazards in the predicted impacts of climate change on human populations. Global Ecology and Biogeography, 20(4), 532-544. https://doi. org/10.1111/j.1466-8238.2010.00632.x

Sjöberg, L. (2000). Factors in risk perception. Risk Analysis, 20(1), 1-12. https://doi.org/10.1111/02724332.00001

Slovic, P. (1992). Perception of risk: Reflections on the psychometric paradigm. En S. Krimsky, \& D. Golding (Eds.), Social theories of risk (pp. 117-152). Praeger.

Smith N., \& Leiserowitz, A. (2014). The role of emotion in global warming policy support and opposition. Risk Analysis, 34, 937-948. 
Spence, A., \& Pidgeon, N. F. (2010). Framing and communicating climate change: the effects of distance and outcome frame manipulations. Global Environmental Change, 20, 656-667.

Swim, J. K., Stern, P. C., Doherty, T. J., Clayton, S., Reser, J. P., Weber, E. U., Gifford, R., \& Howard, G. S. (2011). Psychology's contributions to understanding and addressing global climate change. American Psychologist, 66(4), 241-250. http://doi. org/10.1037/a0023220

van der Linden, S. (2015). The social-psychological determinants of climate change risk perceptions: towards a comprehensive model. Journal of Environmental Psychology, 41, 112-124. http:// dx.doi.org/10.1016/j.jenvp.2014.11.012.
Weber, E. U. (2010). What shapes perceptions of climate change? Wiley Interdisciplinary Reviews: Climate Change, 1(3), 332-342. https://doi.org/10.1002/ wcc. 41

\section{Correspondencia:}

fredy.monge@unsaac.edu.pe

Recibido: 16/04/2021

Aceptado: 16/06/2021 